\title{
A NON-HYPERELLIPTIC CURVE WITH TORSION CERESA CYCLE MODULO ALGEBRAIC EQUIVALENCE
}

\author{
ARNAUD BEAUVILLE AND CHAD SCHOEN
}

\begin{abstract}
We exhibit a non-hyperelliptic curve $C$ of genus 3 such that the class of the Ceresa cycle $[C]-\left[C^{-}\right]$in $J C$ modulo algebraic equivalence is torsion.
\end{abstract}

\section{INTRODUCTION}

Let $C$ be a complex curve of genus $g \geq 2$, and $p$ a point of $C$. We embed $C$ into its Jacobian $J$ by the Abel-Jacobi map $x \mapsto[x]-[p]$; we denote by $C^{-}$the image of $C$ under the involution $(-1)_{J}: a \mapsto-a$ of $J$. The Ceresa class is the class $\mathfrak{z}(C):=[C]-\left[C^{-}\right]$in the group $A_{1}(J)$ of 1 -cycles on $J$ modulo algebraic equivalence (it is independent of the choice of $p$ ). Since $(-1)_{J}$ acts trivially on $H^{p}(J, \mathbb{Z})$ for $p$ even, $\mathfrak{z}(C)$ belongs to the Griffiths group $G(J)$, the kernel of the cycle class map $A_{1}(J) \rightarrow H^{2 g-2}(J, \mathbb{Z})$.

Ceresa classes have played a prominent role in the study of Griffiths groups, especially in the development of techniques for showing that a given element is non-zero [C, C-P,, $\mathrm{H}]$. In addition they played an important role in showing that $G(J)$ can have infinite rank [N]. As the conjectures of Bloch and Beilinson were developed and are studied $\mathfrak{z}(C)$ appears repeatedly [B], BST], [Z, §1.5], always as an element of infinite order.

When $C$ is hyperelliptic, $\mathfrak{z}(C)=0$; in fact $C-C^{-}$is zero as a cycle when $p$ is a Weierstrass point. In this note we will exhibit what we believe to be the first example of a non-hyperelliptic curve $C$ with $\mathfrak{z}(C)=0$ in $A_{1}(J) \otimes \mathbb{Q}$. The curve $C$ has genus 3 , and admits an automorphism $\sigma$ of order 9 , such that the quotient variety $J /\langle\sigma\rangle$ is uniruled. This implies that the Griffiths group of a resolution of $J /\langle\sigma\rangle$ is torsion; going back to $J$ gives the result.

\section{MAIN RESULT}

Theorem. Let $C \subset \mathbb{P}^{2}$ be the genus 3 curve defined by $X^{4}+X Z^{3}+Y^{3} Z=0$. Then $\mathfrak{z}(C)=0$ in $A_{1}(J) \otimes \mathbb{Q}$.

Proof: Let $\zeta$ be a primitive 9-th root of unity. We consider the automorphism $\sigma$ of $C$ defined by $\sigma(X, Y, Z)=\left(X, \zeta^{2} Y, \zeta^{3} Z\right)$. We use the fixed point $p=(0,0,1)$ to embed $C$ in its Jacobian $J$, so that the action of $\sigma$ on $J$ preserves $C$ and $C^{-}$. We denote by $V$ the quotient variety $J /\langle\sigma\rangle$, and by $\pi: J \rightarrow V$ the quotient map. Let $F \subset J$ be the subset of elements with nontrivial stabilizer; the singular locus $\operatorname{Sing} V$ of $V$ is $\pi(F)$. We put $J^{\circ}:=J \backslash F$ and $V^{\circ}:=V \backslash \operatorname{Sing} V$.

Lemma 1. Sing $V$ is finite; the points $\pi(x)$ for $x \in \operatorname{Ker}\left(1_{J}-\sigma\right)$ are non-canonical singularities.

Proof: The space $T_{0}(J)$ is canonically identified with $H^{0}\left(C, K_{C}\right)^{*}$. The elements of $H^{0}\left(C, K_{C}\right)$ are of the form $L \frac{X d Z-Z d X}{Y^{2} Z}$, with $L \in H^{0}\left(\mathbb{P}^{2}, \mathcal{O}_{\mathbb{P}}(1)\right)$ [B-K] $\S 9.3$, Corollary of Theorem 1]. It follows that the eigenvalues of $\sigma$ on $H^{0}\left(C, K_{C}\right)$ are $\zeta^{5}, \zeta^{7}, \zeta^{8}$, and those on $T_{0}(J)=H^{0}\left(C, K_{C}\right)^{*}$ are $\zeta, \zeta^{2}, \zeta^{4}$.

Thanks to E. Colombo and B. van Geemen for their crucial input. The second named author thanks S. Katz and M. Reid for helpful discussions. 
Therefore $\operatorname{Ker}\left(1_{J}-\sigma^{d}\right)$ is finite for $0<d<9$, so $F$ is finite. Since $1+2+4<9$, Reid's criterion [R, Theorem 3.1] implies that the singular points $\pi(x)$ for $x \in \operatorname{Ker}\left(1_{J}-\sigma\right)$ are not canonical.

Lemma 2. The variety $V$ is uniruled.

Proof : Let $\rho: \tilde{V} \rightarrow V$ be a resolution of singularities; it suffices to prove that $\tilde{V}$ has Kodaira dimension $-\infty[\overline{\mathrm{M}}]$. Suppose this is not the case: there exist an integer $r \geq 1$ and a nonzero section $\tilde{\omega}$ of $K_{\tilde{V}}^{r}$. By restriction to $\rho^{-1}\left(V^{\mathrm{o}}\right) \cong V^{\mathrm{o}}$, we get a section $\omega$ of $K_{V^{\circ}}^{r}$, whose pull back under $\pi$ is a nonzero section of $K_{J^{\circ}}^{r}$; therefore $\omega$ is a generator of $K_{V^{\circ}}^{r}$, hence extends to a generator of the reflexive sheaf $K_{V}^{[r]}$ (with the notation of $[\mathbb{R}]$ ). By construction this generator remains regular on $\tilde{V}$, which means that the singularities of $V$ are canonical [ $\mathbb{R}$, Proposition 1.2], contradicting Lemma 1 .

Lemma 3. Let $X$ be a uniruled smooth projective threefold. The Griffiths group $G(X)$ is torsion.

Proof: There exists a smooth projective surface $S$ and a dominant rational map $S \times \mathbb{P}^{1} \rightarrow X$. After blowing up some points and some smooth curves in $S \times \mathbb{P}^{1}$, we get a smooth projective threefold $W$ birational to $S \times \mathbb{P}^{1}$, and a generically finite morphism $f: W \rightarrow X$. Since the Griffiths group is a stably birational invariant (see [V] Proposition 2.30]), we have $G(W)=G(S)=0$. For $z \in G(X)$, we have $(\operatorname{deg} f) z=f_{*} f^{*}(z)=0$, hence $G(X)$ is annihilated by $\operatorname{deg} f$.

Remark.- One can actually deduce from [Bl-S, Theorem 1 (ii)] that $G(X)=0$ - but we will not need this fact.

Proof of the Theorem: We can choose the resolution $\rho: \tilde{V} \rightarrow V$ so that $E:=\rho^{-1}(\operatorname{SingV})$ is a normal crossing divisor, whose irreducible components are smooth and rational [Fuj], Corollary of Theorem $1]$.

Let $\bar{C}$ and $\bar{C}^{-}$be the images in $V$ of $C$ and $C^{-}$, and let $\tilde{C}$ and $\tilde{C}^{-}$be their proper transforms in $\tilde{V}$. We have $[\bar{C}]-\left[\bar{C}^{-}\right]=\frac{1}{9} \pi_{*}\left([C]-\left[C^{-}\right]\right)=0$ in $H^{4}\left(V^{\mathrm{o}}, \mathbb{Q}\right)$. Now we have an exact sequence [D, Corollaire 8.2.8]

$$
H^{2}(\tilde{E}, \mathbb{Q}) \stackrel{i_{*}}{\longrightarrow} H^{4}(\tilde{V}, \mathbb{Q}) \rightarrow H^{4}\left(V^{\mathrm{o}}, \mathbb{Q}\right),
$$

where $\tilde{E}$ is the normalization of $E$, and $i$ the composition $\tilde{E} \rightarrow E \hookrightarrow \tilde{V}$. Therefore we have $[\tilde{C}]-\left[\tilde{C}^{-}\right]=i_{*} z$ in $H^{4}(\tilde{V}, \mathbb{Q})$ for some class $z \in H^{2}(\tilde{E}, \mathbb{Q})$. Since the components of $\tilde{E}$ are rational, $z$ is the class of an element $\mathfrak{z}$ of $A_{1}(\tilde{E}) \otimes \mathbb{Q}$. Then $[\tilde{C}]-\left[\tilde{C}^{-}\right]-i_{* \mathfrak{z}} \in A_{1}(\tilde{V}) \otimes \mathbb{Q}$ is homologous to zero, hence equal to zero by Lemma 3 , Restricting to $\tilde{V} \backslash E \cong V^{\text {o }}$, we get $[\bar{C}]-\left[\bar{C}^{-}\right]=0$ in $A_{1}\left(V^{\mathrm{o}} \otimes \mathbb{Q}\right)$, hence $[C]-\left[C^{-}\right]=\pi^{*}\left([\bar{C}]-\left[\bar{C}^{-}\right]\right)=0$ in $A_{1}\left(J^{\circ}\right) \otimes \mathbb{Q}$. But the restriction map $A_{1}(J) \rightarrow A_{1}\left(J^{\circ}\right)$ is an isomorphism [Ful, Example 10.3.4], hence the Theorem.

\section{COMPLEMENTS}

Corollary 1. Let $\Theta$ be a Theta divisor on $J$. We have $[C]=\frac{[\Theta]^{2}}{2}$ in $A_{1}(J) \otimes \mathbb{Q}$ (Poincaré formula).

Proof: Indeed for any genus 3 curve $C$ we have $[\Theta]^{2}=[C]+\left[C^{-}\right]$in $A_{1}(J) \otimes \mathbb{Q}$ (if $p, q$ are two distinct points of $C$, the intersection of $\Theta$ with its translate by $[p]-[q]$ is the union of a translate of $C$ and a translate of $C^{-}$- see for instance [Mu, Lecture IV]). Thus the corollary is equivalent to the theorem.

Recall that the modified diagonal cycle $\Gamma(C, p)$, first considered in [G-S], is the element $\Gamma(C, p)$ of $A_{1}\left(C^{3}\right)$ defined as follows. We denote by $[x, x, x],[x, x, p],[x, p, p]$ etc. the classes in $A_{1}(C \times C \times C)$ of the image of $C$ by the maps $x \mapsto(x, x, x), x \mapsto(x, x, p), x \mapsto(x, p, p)$ etc. Then:

$$
\Gamma(C, p):=[x, x, x]-[x, x, p]-[x, p, x]-[p, x, x]+[x, p, p]+[p, x, p]+[p, p, x] .
$$

By [FLV], Remark 3.4], we have 
Corollary 2. $\Gamma(C, p)=0$ in $A_{1}\left(C^{3}\right) \otimes \mathbb{Q}$.

Finally let us mention the result of [B3]: the class of $[C]-\left[C^{-}\right]$in the intermediate Jacobian $\mathscr{J}_{1}(J)$ is torsion. It can be also deduced from our theorem, though the proof in [B3] is more direct.

In [BLLS] the authors construct a genus 7 curve with the same property, and suggest that the corresponding Ceresa cycle should be torsion modulo algebraic equivalence (Remark 1.2).

\section{REFERENCES}

[B1] A. Beauville: Sur l'anneau de Chow d'une variété abélienne. Math. Ann. 273 (1986), 647-651.

[B2] A. Beauville: Algebraic cycles on Jacobian varieties. Compositio Math. 140 (2004), 683-688.

[B3] A. Beauville: A non-hyperelliptic curve with torsion Ceresa class. C.R. Acad. Sci. Paris, to appear; preprint arXiv:2105.07160.

[BLLS] D. Bisogno, W. Li, D. Litt, P. Srinivasan: Group-theoretic Johnson classes and non-hyperelliptic curves with torsion Ceresa class. Preprint arXiv:2004.06146.

[Bl] S. Bloch: Algebraic cycles and values of L-functions. J. reine angew. Math. 350 (1984), 94-107.

[Bl-S] S. Bloch, V. Srinivas: Remarks on correspondences and algebraic cycles. Amer. J. Math. 105 (1983), no. 5, 1235-1253.

[B-K] E. Brieskorn, H. Knörrer : Plane algebraic curves. Birkhäuser Verlag, Basel, 1986.

[BST] J. Buhler, C. Schoen, J. Top: Cycles, L-functions and triple products of elliptic curves. J. Reine Angew. Math. 492 (1997), 93-133.

[C] G. Ceresa : $C$ is not algebraically equivalent to $C^{-}$in its Jacobian. Ann. of Math. 117 (1983), no. 2, $285-291$.

[C-vG] E. Colombo, B. van Geemen: Note on curves in a Jacobian. Compositio Math. 88 (1993), 333-353.

[C-P] A. Collino, G. P. Pirola : The Griffiths infinitesimal invariant for a curve in its Jacobian. Duke Math J. 78 (1995), $59-88$.

[D] P. Deligne: Théorie de Hodge, III. Publ. Math. IHES 44 (1974), 5-77.

[Fuj] A. Fujiki : On resolutions of cyclic quotient singularities. Publ. Res. Inst. Math. Sci. 10 (1974/75), no. 1, $293-328$.

[Ful] W. Fulton: Intersection theory. Ergebnisse der Math. 2. Springer-Verlag, Berlin, 1984.

[FLV] L. Fu, R. Laterveer, C. Vial: Multiplicative Chow-Künneth decompositions and varieties of cohomological K3 type. Ann. Mat. Pura Appl. 200 (2021), no. 5, p. 2085-2126.

[G-S] B. Gross, C. Schoen: The modified diagonal cycle on the triple product of a pointed curve. Ann. Inst. Fourier 45 (1995), 649-679.

[H] B. Harris : Homological versus algebraic equivalence in a Jacobian. Proc. Nat. Acad. Sci. U.S.A. 80 (1983), no. 4, 11571158.

[M] Y. Miyaoka: On the Kodaira dimension of minimal threefolds. Math. Ann. 281 (1988), 325-332.

$[\mathrm{Mu}]$ D. Mumford: Curves and their Jacobians. The red book of varieties and schemes, Lecture Notes in Math. 1358. Springer-Verlag, Berlin, 1999.

[N] M. Nori: Cycles on the generic abelian threefold. Proc. Indian Acad. Sci. Math. Sci. 99 (1989), no. 3, 191-196.

[R] M. Reid: Canonical 3-folds. Journées de Géometrie Algébrique d'Angers, pp. 273-310, Sijthoff \& Noordhoff, 1980.

[V] C. Voisin: Birational invariants and decomposition of the diagonal. Birational Geometry of Hypersurfaces, pp. 3-71; Lecture Notes of the UMI 26, Springer, 2019.

[Z] S-W Zhang: Gross-Schoen cycles and dualising sheaves. Invent. math. 179 (2010), no. 1, 1-73.

Université Côte D'Azur, CNRS - Laboratoire J.-A. Dieudonné, Parc Valrose, F-06108 Nice Cedex 2, France

Email address: arnaud.beauville@unice.fr

Department of Mathematics, Duke University Box 90320, Durham NC 27708-0320, USA

Email address: schoen@math.duke.edu 